\title{
PAPER
}

\section{Free evolution of a mixture of quantum states with classical space and} momentum uncertainties

To cite this article: R O Barrachina et al 2019 Eur. J. Phys. 40065402

View the article online for updates and enhancements.

\section{IOP ebooks}

Bringing you innovative digital publishing with leading voices

to create your essential collection of books in STEM research.

Start exploring the collection - download the first chapter of every title for free. 


\title{
Free evolution of a mixture of quantum states with classical space and momentum uncertainties
}

\author{
R O Barrachina ${ }^{1,2}$ (1), F Navarrete ${ }^{3}$ (1) and M F Ciappina ${ }^{4}$ (1) \\ ${ }^{1}$ Centro Atómico Bariloche and Instituto Balseiro, Comisión Nacional de Energía \\ Atómica (CNEA) and Universidad Nacional de Cuyo, Av. Bustillo 9500, 8400 \\ Bariloche, Argentina \\ ${ }^{2}$ Consejo Nacional de Investigaciones Científicas y Técnicas (CONICET), Argentina \\ ${ }^{3}$ Department of Physics, Kansas State University, Manhattan, Kansas 66506, United \\ States of America \\ ${ }^{4}$ Institute of Physics of the ASCR, ELI-Beamlines, Na Slovance 2, 18221 Prague, \\ Czech Republic \\ E-mail: barra@cab.cnea.gov.ar
}

Received 27 June 2019, revised 7 August 2019

Accepted for publication 15 August 2019

Published 18 September 2019

\begin{abstract}
This article continues and completes a recent one regarding a quantum version of van Cittert-Zernike's theorem (Fabre et al 2018 Eur. J. Phys. 39 015401). In that paper, we considered a mixture of quantum states that only differed in their initial positions, but not on their momenta. Here, we relax this nonphysical assumption and analyse how the inclusion of both classical uncertainties in the initial position and momentum of a mixture of — otherwise identical—free quantum states which do not interact with an environment, affects its quantum coherence length. We show that the mathematical development and the final result are simple enough to make possible the incorporation of this subject into a basic course of quantum physics. Furthermore, we demonstrate that these results provide a comprehensive solution to a recent controversy in atomic collision physics.
\end{abstract}

Keywords: quantum coherence, coherence length, density matrix

(Some figures may appear in colour only in the online journal)

\section{Introduction}

What are we talking about when we talk about quantum coherence? If we rely on the bestknown quantum mechanics textbooks, it is hard to say. Except for a few exceptions, the 
word 'coherence' does not even appear in the alphabetical indexes of the vast majority of them.

Perhaps, the reason for the absence of this topic in the standard syllabus of most introductory courses is mainly pedagogical. The transition from classical to quantum physics represents such a gigantic paradigm shift that most textbooks are content to focus only on the study of pure states. Addressing the subtleties of mixed states would be an additional complication that might be better to avoid or, rather, to postpone for a more advanced course or to one specifically dealing with quantum statistical physics.

Sound as this approach might be from a didactic point of view, the problem is that in the real world quantum effects and experiments rarely deal with pure states. Take, for example, Young's two-slit experiment with which Feynman starts the third volume of his famous Physics Lectures (Feynman et al 2010). It is very unlikely that each and every electron emitted towards the slits could be represented by the same wave function. For instance, the electrons can have slightly different velocities, or might be emitted from different positions of the 'macroscopic' electron gun. And no matter how much we struggle to reduce these classical uncertainties, we could never narrow them enough to suppose them null. In other words, we would never be able to describe all electrons emitted from that 'electron gun' by a single and unique wave function.

Some textbooks (e.g. Ballentine 2000, Messiah 2014) explain the concept of a 'density operator' as a way of incorporating classical statistics into the description of real quantum systems, but they are the exceptions. However, this formalism is simple enough to be introduced in a basic course, and opens the door to a full and easily understandable description of coherence.

In a previous article (Fabre et al 2018) we employed some very basic ideas from the density operator formalism to provide an intuitive explanation of what quantum coherence is. Not only did we manage to introduce an operational definition of the coherence length (i.e. a definition based upon what can be measured), but we could also give a clear and precise meaning to van Cittert-Zernike's theorem (van Cittert 1934, Zernike 1938), explaining how an incoherent mixture of states can increase its coherence as time evolves.

In that article we assumed that the quantum states making up the mixture could only differ in their initial positions; but chances are that they would also have different initial momenta, both in direction and magnitude. In the present article we investigate how this classical uncertainty in the momentum might modify the coherence length and its time evolution. In this way we provide a complete description of how the classical uncertainties in position and momentum affect the coherence of a mixture of quantum states.

This article is organized as follows. In section 2 we review some results from our previous article (Fabre et al 2018) related to the time evolution of a free quantum state. In the following section we study an ensemble of pure states which differ in their initial position and momentum, and define its density operator. It is important to point out that the coherence that we discuss in this article is related to the free evolution of a quantum mixture which does not interact with any environment. As a working example, in section 4 we apply these results to the case of Gaussian probabilities and wave functions. In section 5 we obtain an analytic expression for the coherence length. In all these results we incorporate the classical uncertainties in both the initial position and impulse, instead of only in the initial position, as we have done in our previous article (Fabre et al 2018). Finally we describe the similarities and differences between both effects, and how they can 
be used to solve a controversy that recently arose in atomic collision physics (see. e.g. Schulz 2017).

\section{Free wave packet}

As in the previous article (Fabre et al 2018), and without any loss of generality, we consider a one-dimensional wave function describing a particle of mass $m$ and momentum $p$ that at time $t=0$ is centered at $b$, namely

$$
\left\langle x \mid \phi_{p, b}(0)\right\rangle=\mathrm{e}^{\mathrm{i} p x / \hbar}\left\langle x-b \mid \phi_{0,0}(0)\right\rangle .
$$

In momentum representation it reads

$$
\left\langle k \mid \phi_{p, b}(0)\right\rangle=\mathrm{e}^{-\mathrm{i}(k-p) b / \hbar}\left\langle k-p \mid \phi_{0,0}(0)\right\rangle .
$$

We are assuming that $\phi_{0,0}(0)$ is a normalized wave function located at the origin with zero group velocity, namely

$$
\begin{aligned}
\left\langle\phi_{0,0}(0) \mid \phi_{0,0}(0)\right\rangle & =1, \\
\left\langle\phi_{0,0}(0)|x| \phi_{0,0}(0)\right\rangle & =0, \\
\left\langle\phi_{0,0}(0)|k| \phi_{0,0}(0)\right\rangle & =0 .
\end{aligned}
$$

The free time evolution of the wave function is trivial in momentum space,

$$
\left\langle k \mid \phi_{p, b}(t)\right\rangle=\mathrm{e}^{-\mathrm{i} E_{k} t / \hbar}\left\langle k \mid \phi_{p, b}(0)\right\rangle,
$$

with $E_{k}=k^{2} / 2 m$. On the other hand, in space representation,

$$
\left\langle x \mid \phi_{p, b}(t)\right\rangle=\frac{1}{\sqrt{2 \pi \hbar}} \int \mathrm{e}^{\mathrm{i}\left(k x-\mathrm{i} E_{k} t\right) / \hbar}\left\langle k \mid \phi_{p, b}(0)\right\rangle \mathrm{d} k .
$$

\section{Incoherent states and density operator}

Let us now consider an ensemble composed of wave functions $\phi_{p, b}(t)$, where the initial position $b$ and momentum $p$ are distributed according to classical probabilities $f(b)$ and $g(p)$, respectively. Due to these uncertainties in the initial position and momentum, it is no longer possible to describe the ensemble with a single wave function. We say that we are dealing with an incoherent mixture of free-evolving wave packets. Each wave evolves as described in equation (5), but starting at different positions $b$ and with different momenta $p$. Thus, it is unavoidable to incorporate the classical probabilities $f(b)$ and $g(p)$ in the evaluation of the mean value of any observable $\mathcal{A}$,

$$
\langle\mathcal{A}\rangle_{t}=\int \mathrm{d} b \mathrm{~d} p\left\langle\phi_{p, b}(t)|\mathcal{A}| \phi_{p, b}(t)\right\rangle f(b) g(p) .
$$

Similarly, the probability distributions of position and momentum read

$$
P(x, t)=\int \mathrm{d} b \mathrm{~d} p\left|\left\langle x \mid \phi_{p, b}(t)\right\rangle\right|^{2} f(b) g(p)
$$

and

$$
\tilde{P}(k, t)=\int \mathrm{d} b \mathrm{~d} p\left|\left\langle k \mid \phi_{p, b}(t)\right\rangle\right|^{2} f(b) g(p) .
$$


Note how we have to incorporate quantum and classical statistics simultaneously, in order to deal with the incomplete knowledge of the initial state. As it was asserted previously (Fabre et al 2018), the uncertainties $\Delta b$ and $\Delta p$ should not be confused with those of the coordinate $\Delta x$ and momentum $\Delta k$ of the individual wave packets. While the previous ones have a classical origin, the latter are of a purely quantum nature.

It is easy to demonstrate that, by defining the density operator (Blum 2012),

$$
\rho=\int \mathrm{d} b \mathrm{~d} p\left|\phi_{p, b}(t)\right\rangle f(b) g(p)\left\langle\phi_{p, b}(t)\right|,
$$

the probability distributions of position and momentum can be written as follows:

$$
P(x, t)=\langle x|\rho| x\rangle \text { and } \tilde{P}(k, t)=\langle k|\rho| k\rangle,
$$

while

$$
\langle\mathcal{A}\rangle_{t}=\int \mathrm{d} x\langle x|\mathcal{A} \rho| x\rangle=\int \mathrm{d} k\langle k|\mathcal{A} \rho| k\rangle
$$

As it was explained in (Fabre et al 2018), the density operator $\rho(t)$ provides a complete and compact description of an incoherent mixture, as a wave function does for a pure system. It evolves according to the Liouville-von Neumann equation,

$$
\frac{\partial \rho}{\partial t}=\frac{\mathrm{i}}{\hbar}\left[\rho, H_{o}\right]
$$

which plays the role of the Schrödinger equation for a pure state. Let us finally give a step forward, and write down the density matrix in space representation,

$$
\begin{aligned}
\left\langle x|\rho| x^{\prime}\right\rangle & =\int \mathrm{d} b \mathrm{~d} p\left\langle x \mid \phi_{p, b}(t)\right\rangle f(b) g(p)\left\langle\phi_{p, b}(t) \mid x^{\prime}\right\rangle \\
& =\int \mathrm{d} b \mathrm{~d} p \mathrm{e}^{\mathrm{i} p\left(x-x^{\prime}\right) / \hbar}\left\langle x-b-p t / m \mid \phi_{0,0}(t)\right\rangle f(b) g(p)\left\langle\phi_{0,0}(t) \mid x^{\prime}-b-p t / m\right\rangle,
\end{aligned}
$$

and in momentum representation,

$$
\begin{aligned}
\left\langle k|\rho| k^{\prime}\right\rangle & =\int \mathrm{d} b \mathrm{~d} p\left\langle k \mid \phi_{p, b}(t)\right\rangle f(b) g(p)\left\langle\phi_{p, b}(t) \mid k^{\prime}\right\rangle \\
& =\mathrm{e}^{-\mathrm{i}\left(E_{k}-E_{k^{\prime}}\right) t / \hbar} \tilde{f}\left(k-k^{\prime}\right) \int \mathrm{d} p\left\langle k-p \mid \phi_{0,0}(0)\right\rangle g(p)\left\langle\phi_{0,0}(0) \mid k^{\prime}-p\right\rangle,
\end{aligned}
$$

with

$$
\tilde{f}(q)=\int \mathrm{d} b f(b) \mathrm{e}^{-\mathrm{i} q b / \hbar}
$$

\section{Gaussian wave and probabilities}

As a working example we will represent the classical probabilities $f(b)$ and $g(p)$, and the wave function $\phi_{0,0}$ by Gaussian functions (Fabre et al 2018):

$$
f(x)=\left(\frac{1}{2 \pi(\Delta b)^{2}}\right)^{1 / 2} \mathrm{e}^{-\left(b-b_{o}\right)^{2} / 2(\Delta b)^{2}}
$$




$$
g(p)=\left(\frac{1}{2 \pi(\Delta p)^{2}}\right)^{1 / 2} \mathrm{e}^{-\left(p-p_{o}\right)^{2} / 2(\Delta p)^{2}}
$$

and

$$
\begin{aligned}
& \left\langle x \mid \phi_{0,0}(t)\right\rangle=\left(\frac{(\Delta x)^{2}}{2 \pi\left[(\Delta x)^{2}+\mathrm{i} \hbar t / 2 m\right]^{2}}\right)^{1 / 4} \mathrm{e}^{-x^{2} / 4\left[(\Delta x)^{2}+\mathrm{i} \hbar t / 2 m\right]}, \\
& \left\langle k \mid \phi_{0,0}(t)\right\rangle=\left(\frac{1}{2 \pi(\Delta k)^{2}}\right)^{1 / 4} \mathrm{e}^{-\mathrm{i} E_{k} t / \hbar} \mathrm{e}^{-k^{2} / 4(\Delta k)^{2}},
\end{aligned}
$$

with $\Delta k=\hbar / 2 \Delta x$. It is important to point out that this choice of Gaussian shapes does not imply a limitation on the general validity of the expression for the coherence length (Fabre et al 2018), as obtained in the following section.

After some lengthy but simple calculations, the corresponding density matrix reads

$$
\left\langle x|\rho| x^{\prime}\right\rangle=\langle x \mid \Phi(t)\rangle \mathrm{e}^{-\left(x-x^{\prime}\right)^{2} / 2 \Lambda^{2}}\left\langle\Phi(t) \mid x^{\prime}\right\rangle,
$$

where

$$
\langle x \mid \Phi(t)\rangle=\mathrm{e}^{\mathrm{i} p_{o} x / \hbar}\left(\frac{\operatorname{Re}\left(\Sigma^{2}\right)}{2 \pi \Sigma^{4}}\right)^{1 / 4} \mathrm{e}^{-\left(x-\langle x\rangle_{t}\right)^{2} / 4 \Sigma^{2}},
$$

with $\langle x\rangle_{t}=b_{o}+p_{o} t / m$,

$$
\Sigma=\sqrt{\frac{\left[(\Delta x)^{2}+(\Delta b)^{2}\right]+t^{2}\left[(\Delta k)^{2}+(\Delta p)^{2}\right] / m^{2}}{1-2 \mathrm{i} t\left[(\Delta k)^{2}+(\Delta p)^{2}\right] / \hbar m}},
$$

and

$$
\Lambda=\sqrt{\frac{\left[(\Delta x)^{2}+(\Delta b)^{2}\right]+t^{2}\left[(\Delta k)^{2}+(\Delta p)^{2}\right] / m^{2}}{\left[(\Delta x)^{2}+(\Delta b)^{2}\right]\left[(\Delta k)^{2}+(\Delta p)^{2}\right] / \hbar^{2}-1 / 4}} .
$$

These results generalize those obtained in a previous article (Fabre et al 2018) for the particular case $\Delta p=0$.

\section{Coherence length}

The fingerprint of a coherent ensemble of particles is given by its ability to produce effects that cannot be described in classical terms The more conspicuous and well-known of these effect is that of 'interference', as exemplified by the two-slit experiment. Now, for this experiment to work, i.e. for an interference pattern to appear, the beam of particles has to 'illuminate' the two slits coherently. Thus, we introduce a so-called coherence length $\ell$ associated to the density operator such that for the interference to be observable, it has to be larger than or at least comparable with the distance between the slits. Otherwise, if $\ell$ is much smaller than the distance between the slits, the particle's beam will at the most 'illuminate' one of the slits, but not the other, and the interference pattern will not be formed. As it should be evident, we are defining $\ell$ operationally as representative of the separation between the slits in a Young experiment beyond which the interference pattern is too feeble to be observed.

Thus, let us evaluate the density matrix element (Fabre et al 2018)

$$
\mathcal{T}=\langle(\langle x\rangle+\delta x / 2)|\rho(t)|(\langle x\rangle-\delta x / 2)\rangle .
$$


It can be easily demonstrated that

$$
\mathcal{T} \propto \exp \left(-(\delta x)^{2} / 2 \ell^{2}\right)
$$

with

$$
\frac{1}{\ell^{2}}=\frac{1}{\Lambda^{2}}+\frac{\operatorname{Re}\left(\Sigma^{2}\right)}{4|\Sigma|^{4}} .
$$

Some straightforward calculations lead to the following simple expression for the coherence length,

$$
\ell_{\Delta p, \Delta b}(t)=\hbar \sqrt{\frac{1}{(\Delta k)^{2}+(\Delta p)^{2}}+\frac{(t / m)^{2}}{(\Delta x)^{2}+(\Delta b)^{2}}} .
$$

\subsection{Pure state}

Let us first note that for $\Delta b=0$ and $\Delta p=0$, we recover the density matrix for a pure state,

$$
\left\langle x|\rho| x^{\prime}\right\rangle=\left\langle x \mid \phi_{p, b}(t)\right\rangle\left\langle\phi_{p, b}(t) \mid x^{\prime}\right\rangle,
$$

with a coherence length

$$
\begin{aligned}
& \ell_{0,0}(t)=\hbar \sqrt{\frac{1}{(\Delta k)^{2}}+\left(\frac{t}{m \Delta x}\right)^{2}} \\
& =2 \sqrt{(\Delta x)^{2}+\left(\frac{\Delta k t}{m}\right)^{2}} .
\end{aligned}
$$

It might be surprising that an ensemble of pure states, which by definition is purely coherent, should have a finite coherence length. But, in fact, $\ell$ is representative of the full spatial width of the wave function, and it has to be larger than the distance between the slits for the interference pattern to appear.

\subsection{Mixed state}

Let us now consider a case where neither $\Delta p$ nor $\Delta b$ are zero. The first thing that strikes the eye when comparing equations (27) and (29) is that they are not so different after all, at least from a formal point of view. We see that $\ell_{\Delta p, \Delta b}$ is still similar to $\ell_{0,0}$ in equation (29), but with $\Delta k$ replaced with $\sqrt{(\Delta k)^{2}+(\Delta p)^{2}}$ and $\Delta x$ with $\sqrt{(\Delta x)^{2}+(\Delta b)^{2}}$. At first glance they seem inconsequential changes, but in reality they are of enormous importance.

To start with, we see that the coherence length of a mixed state is dramatically reduced with respect to that of a pure state, i.e. $\ell_{\Delta p, \Delta b} \ll \ell_{0,0}$. But this was, of course, a predictable result of the introduction of classical (and therefore macroscopic) uncertainties. On the contrary, the fact that the mixed state still shows a certain level of coherence and, above all, that $\ell_{\Delta p, \Delta b}$ increases with time can be quite surprising. In particular, for very large values of $t$, we obtain

$$
\ell_{\Delta p, \Delta b}(t) \approx \frac{\hbar t / m}{\sqrt{(\Delta x)^{2}+(\Delta b)^{2}}} .
$$

This result represents the van Cittert-Zernike effect of optics applied to quantum particles. For instance, in optics, the light emitted by a source as obviously incoherent as the Sun 


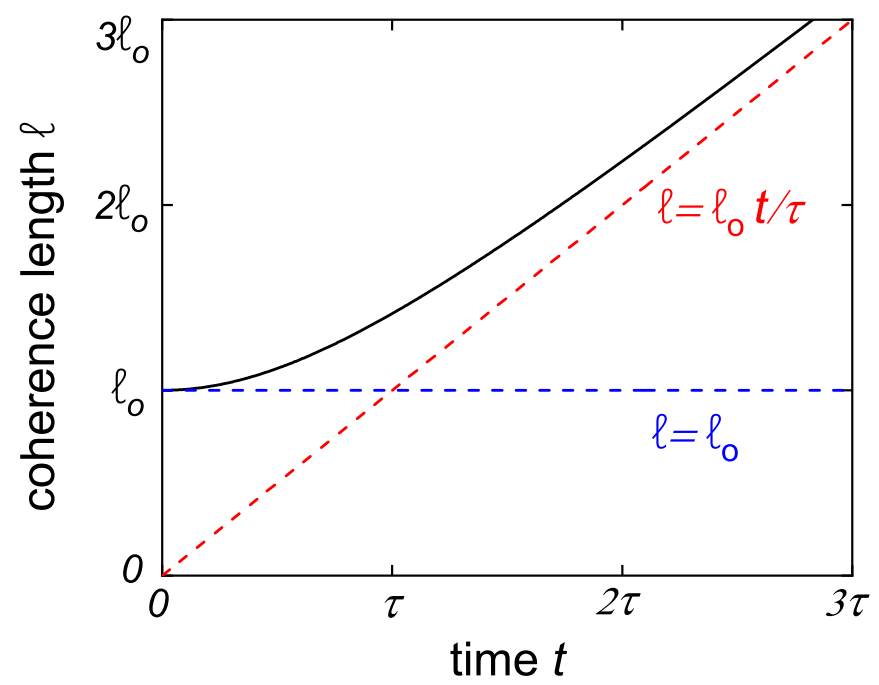

Figure 1. The time evolution of the coherence length $\ell$ is compared with its Fresnel $\left(\ell \approx \ell_{o}\right)$ and Fraunhofer $\left(\ell=\ell_{o} t / \tau\right)$ limits. The characteristic length $\ell_{o}$ and time $\tau$ are defined in the text.

presents-upon reaching the Earth - a coherence length of some tens of $\mu \mathrm{m}$, large enough for Thomas Young to carry out his famous experiment. If we consider the light arriving to Earth from the Alpha Centauri System, located at 4.37 light-years from the Sun, the coherence length is of the order of some few meters. We will not explore this effect any further since it was far more comprehensively discussed in our previous article (Fabre et al 2018).

Note that in equation (31), $\Delta p$ plays no role whatsoever. This result clearly shows that the effects produced by the macroscopic uncertainties $\Delta b$ and $\Delta p$, are far from being similar. While $\Delta p$ only affects the 'minimum' coherence length,

$$
\ell_{o}=\frac{\hbar}{\sqrt{(\Delta k)^{2}+(\Delta p)^{2}}},
$$

$\Delta b$ acts on the increasing rate of the van Cittert-Zernike effect, namely

$$
\frac{\ell_{o}}{\tau}=\frac{\hbar / m}{\sqrt{(\Delta x)^{2}+(\Delta b)^{2}}} .
$$

Thus, while the first one is important in the Fresnel limit (i.e. for $t \approx 0$ ), the second one is strongly dominant for very large times (Fraunhofer limit). This is shown in figure 1, where the general expression for the coherence length,

$$
\ell=\ell_{o} \sqrt{1+\frac{t^{2}}{\tau^{2}}},
$$

interpolates between the Fresnel $\left(\ell \approx \ell_{o}\right)$ and Fraunhofer $\left(\ell=\ell_{o} t / \tau\right)$ limits.

\section{A simple application in atomic and molecular collision physics}

Let us imagine a typical scattering experiment where a beam of charged projectiles (i.e. electrons, ions, etc) collides with a gaseous target consisting of atoms or molecules. A usual 
but tacit assumption of practically all studies of this type of process is that the coherence length of the incident beam is much larger than any characteristic size $a$ of each individual target. In this way, the collision problem can be analysed as if each projectile could be represented by a plane wave (Taylor 2006). However, as it is explained by Schulz (2017), some recent studies, starting with the pioneering one by Egodapitiya et al (2011), show that it is not always true that the coherence length is larger than $a$, and that it can be even manipulated to switch from a purely coherent $(\ell>a)$ to a partially coherent $(\ell \leqslant a)$ or even an incoherent $(\ell \ll a)$ case.

The basic idea behind this disruptive proposal is that not only the uncertainties in position $\Delta b$ and momentum $\Delta p$ (both assumed to be transverse to the direction of movement of the beam) could be controlled by means of collimators and electromagnetic fields, but also-and far more important - that the time $t$ could be modified by changing the distance $L=t p_{0} / m$ travelled by the projectiles until reaching the target.

Now, in any practical situation, we might assume that the width of the collimator, $\Delta b$, being macroscopic is always much larger than the microscopic width $\Delta x$ of each individual wave packet. Thus,

$$
\ell \approx \hbar \sqrt{\frac{1}{(\Delta k)^{2}+(\Delta p)^{2}}+\left(\frac{L}{p_{0} \Delta b}\right)^{2}} .
$$

Surprisingly enough, the comparison between $\Delta k$ and $\Delta p$ is not so straightforward. On one hand, it can be easily shown that $\Delta k / p_{0}$ is of the order of the inverse of the projectile's velocity $v_{0}$ in atomic units (Drake 2006), which in itself is limited by the velocity of light, namely $v_{0}<137$ atomic units. On the other hand, the angular divergence of the ion beam, $\Delta \theta \approx 2 \Delta p / p_{0}$, is usually ranging in the order of the mrad or even less (Schulz 2017). Thus, it is valid to assume that in most practical cases $\Delta \theta \ll 2 \Delta k / p_{0}$, and therefore

$$
\ell \approx \hbar \sqrt{\frac{1}{(\Delta k)^{2}}+\left(\frac{L}{p_{0} \Delta b}\right)^{2}} .
$$

These results are of the utmost importance, since they indicate that while the variation of $L$ represents an effective method to modify the coherence length, the angular divergence would not play any relevant role. In this way, a controversy is answered as to whether both methods can be used equivalently or not to control the coherence length (Gatzke et al 2017).

\section{Conclusions}

In this article we have discussed the effects that the uncertainties (of classical origin) in the position and the initial impulse of the quantum states might produce on the coherence of an ensemble of particles. In this sense, this article is complementary to a previous one (Fabre et al 2018), dedicated to the study of the van Cittert-Zernike effect for the case where only a classical distribution of the initial position was considered, without taking into account possible uncertainties in the impulse.

We have focussed our attention on how the coherence length of a mixture of particles depends on the classical uncertainties in position and momentum. It is important to point out that both the mathematics and the theoretical description are simple enough to be incorporated into a basic course in quantum mechanics. Finally, we showed an application of these results in the field of atomic collisions. 


\section{Acknowledgments}

This work was supported by the project 'Advanced research using high intensity laser produced photons and particles' (CZ.02.1.01/0.0/0.0/16_019/0000789) from the European Regional Development Fund (ADONIS). FN acknowledges the hospitality of the James R Macdonald Laboratory, Department of Physics, Kansas State University, Manhattan, Kansas, USA.

\section{ORCID iDs}

R O Barrachina $\odot$ https://orcid.org/0000-0002-2404-8311

F Navarrete (1) https://orcid.org/0000-0003-4759-4005

M F Ciappina $\odot$ https://orcid.org/0000-0002-1123-6460

\section{References}

Ballentine L E 2000 Quantum Mechanics, A Modern Development (Singapore: World Scientific)

Blum K 2012 Density Matrix Theory and Applications (Berlin: Springer)

Drake G W F 2006 Springer Handbook of Atomic, Molecular, and Optical Physics (Berlin: Springer) p 5

Egodapitiya K N, Sharma S, Hasan A, Laforge A C, Madison D H, Moshammer R and Schulz M 2011 Phys. Rev. Lett. 106153202

Fabre I, Navarrete F, Sarkadi L and Barrachina R O 2018 Eur. J. Phys. 39015401

Feynman R, Leighton R and Sands M 2010 The Feynman Lectures on Physics, Quantum Mechanics vol 3 (Reading, MA: Addison-Wesley)

Gatzke J et al 2017 J. Phys.: Conf. Series 875092006

Messiah A 2014 Quantum Mechanics (Mineola, NY: Dover)

Schulz M 2017 Adv. Atom. Mol. Opt. Phys. 66507

Taylor J R 2006 Scattering Theory: The Quantum Theory of Nonrelativistic Collisions (New York: Dover)

van Cittert P H 1934 Physica 1201

Zernike F 1938 Physica 5785 